\title{
Hogares, alimentación y salarios: La encuesta higiénico- económica del Instituto Nacional de la Nutrición
}

\section{Households, food and wages: The hygienic-economic survey of the "Instituto Nacional de la Nutrición"}

\author{
Paula Lucía Aguilar \\ Consejo Nacional de Investigaciones Científicas y Técnicas \\ Instituto de Investigaciones Gino Germani \\ Universidad de Buenos Aires \\ (Argentina) \\ aguilarpl@gmail.com
}

Entre 1936 y 1937, un conjunto de "Investigadoras Sociales" egresadas del Museo Social Argentino y entrenadas por técnicos del Instituto Nacional de la Nutrición registró durante 31 días los datos de 600 familias de distintos barrios de la Ciudad de Buenos Aires. El relevamiento abordó la composición familiar, su salud, el trabajo de sus integrantes, el presupuesto familiar, la vivienda y muy especialmente el consumo de alimentos. Diversas instituciones de asistencia social tanto públicas como privadas facilitaron el acceso a las familias entrevistadas y colaboraron en el seguimiento de la encuesta. Sus resultados fueron sistematizados por el personal del instituto, difundidos en su Boletín y presentados ante foros de expertos internacionales en diversos problemas sociales. Este trabajo se propone analizar este registro detallado de la situación social -que toma al núcleo familiar como su principal unidad de análisis y reflexión-, e inscribirlo en una serie de iniciativas que, tanto a nivel local como trasnacional, promovían la producción de conocimiento sobre los hogares de las clases trabajadoras, sus presupuestos familiares y sus hábitos de consumo en relación con el salario. Asimismo, entendemos que las categorías y técnicas puestas en juego en estos modos de registro contribuyeron, no sin resistencias, a la delimitación del hogar como espacio de intervención por parte del Estado.

Palabras Clave: Cuestión Social - Domesticidad - Consumo - Políticas Sociales - Salarios 


\begin{abstract}
Between 1936 and 1937, a group of "social researchers" graduated at the "Museo Social Argentino" and trained by experts from the "Instituto Nacional de la Nutrición" collected detailed data from 600 families from different neighborhoods in Buenos Aires during 31 days. The survey addressed the family composition, health, labor situation, and their family budget, especially housing and food consumption rates. Many social assistance institutions, both public and private, facilitated access to the interviewed families and assisted in monitoring the survey. The results obtained were systematized by the institute staff, published in its Bulletin and presented to international experts' forums on diverse social problems. This paper analyzes the detailed record of the work and living conditions -which focused on the family as the main unit of analysis- and enrolls it in a series of initiatives. These initiatives promoted the production of knowledge about working class household, family budgets and consumption habits in relation to salary, both locally and transnationally. We also understand that the categories and techniques at play in these kind of surveys contributed, not without conflict, to the delimitation of household as a place of State intervention.
\end{abstract}

Key Words: Social Question - Domesticity - Consumption - Social Policy - Wages

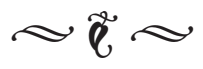

\section{Introducción}

No existe un país tan rico que pueda sufragar los gastos que exige la alimentación permanente de los que no alcanzan a comer suficientemente; ni es posible desentenderse de la solución de este grave problema técnico, económico y social sin peligro de ir al caos. ${ }^{1}$

En los últimos años el campo de investigaciones sobre consumo ha cobrado creciente vitalidad en la Argentina. Investigaciones historiográficas recientes

1. ESCUDERO, Pedro, La politica Nacional de Alimentación en la República Argentina, Ministerio de Relaciones Exteriores y Culto, Instituto Nacional de la Nutrición, Buenos Aires, 1939, p. 1. abordan su estudio para comprender la extensión del bienestar y la modernización de las pautas culturales de los trabajadores durante el siglo XX, analizar aspectos de la cultura material y de la vida cotidiana desde una perspectiva de género; o bien describir los procesos de construcción del consumidor como figura clave en la orientación de las prácticas publicitarias y políticas económicas. ${ }^{2}$ En este

2. ROCCHI, Fernando, "Inventando la soberanía del consumidor: publicidad, privacidad y revolución en Argentina 1860-1940", en DEVOTO, Fernando y MADERO, Marta (directores) Historia de la vida privada en la Argentina. T. II: La Argentina plural, 18701930, Taurus, Buenos Aires 1999; ELENA, Eduardo, Dignifying Argentina. Peronism, citizenship, and mass consumption, University of Pittsburgh Press, Pittsburgh, 2011; MILANESIO, Natalia, Cuando los trabajadores salieron de compras. Nuevos consumidores, publicidad y cambio cultural durante el primer peronismo, Siglo XXI 
contexto, el trabajo que aquí presentamos explora una experiencia singular de producción de información estatal que permite iluminar tanto la relación entre la configuración de la domesticidad y las intervenciones de la política social, como los modos en que se registran y caracterizan los consumos de las clases trabajadoras y se definen pautas de acción sugeridas para su orientación en pos de mejorar sus condiciones de vida.

Durante la década de 1930, numerosas iniciativas de registro sociográfico, contribuyeron a la delimitación de los hogares como objetos de conocimiento, configurando su inteligibilidad para la intervención estatal. Tanto la información presentada en los informes técnicos resultantes, como los diagnósticos y respuestas orientadoras de posibles cursos de acción de gobierno presentes en ellos, dan cuenta de modos específicos de problematización de las condiciones de vida y trabajo de la población. La unidad de análisis fundamental de estos estudios era la familia, con especial interés en la caracterización exhaustiva de la llamada "familia obrera", sus ingresos y consumos.

En octubre de 1939, en ocasión de realizarse en la ciudad de Buenos Aires la Tercera Conferencia Internacional de la alimentación, el Dr. Pedro Escudero ${ }^{3}$, director del Instituto

Editores, Buenos Aires, 2014; PEREZ, Inés, El hogar tecnificado. Familias, género y vida cotidiana (1940-1970), Editorial Biblos, Buenos Aires, 2012; CALDO, Paula, "Recetas, ecónomas, marcas y publicidades: la educación de las mujeres cocineras de la sociedad de consumo (Argentina, 1920-1945)", en Arenal, Granada, 2013, V. 20, № 1, pp. 159-190.

3. Pedro Escudero (1887-1963). Médico egresado en 1902. Profesor de Clínica Médica y de Clínica de la Nutrición de la Facultad de Ciencias Médicas de la Universidad de Buenos Aires (1921). Fundador y primer
Nacional de la Nutrición, presentó ante los expertos, delegados y observadores allí congregados ${ }^{4}$ los principales resultados de la encuesta sobre la "Relación entre el estado económico de la familia, el tipo de alimentación y la salud". ${ }^{5}$ El trabajo compilaba datos detallados de la vida cotidiana de 600 familias de la ciudad de Buenos Aires relevados durante un mes completo. Esta encuesta formaba parte de un conjunto más amplio de estudios oficiales acerca de la situación alimentaria de las poblaciones en distintos países, impulsados por la Sociedad de las Naciones y llevados a cabo durante la segunda mitad de la década de 1930. Las iniciativas de registro contaron además con la colaboración de la Oficina Sanitaria de la Unión Panamericana y fueron apoyadas por la Organización Internacional del Trabajo. ${ }^{6}$

director general del Instituto Nacional de la Nutrición (1938). Presidente de la Asociación Médica Argentina (1912-1922). Miembro de la Academia Nacional de Medicina (1928). Fundador de la Asociación Argentina de Nutrición y Dietología (1941). Es considerado el pionero de la disciplina en América Latina. Datos Biográficos tomados de MARICHAL, Eugenia, Conocimiento científico-técnico y regulaciones jurídicas. El control de los alimentos como red: el caso del Código Bromatológico y el Instituto Bromatológico de la Provincia de Santa Fe (1939-1941), Maestría en Ciencia, Tecnología y Sociedad. Universidad Nacional de Quilmes, 2012, mimeo.

4. Según consta en las actas preliminares de la conferencia, participan en ella delegados de la Sociedad de las Naciones, Bolivia, Brasil, Chile, Colombia, Cuba, Ecuador, EEUU, Méjico, Perú, Uruguay y Venezuela. Asisten en calidad de observadores expertos de Guatemala, Honduras, República Dominicana y Paraguay.

5. ESCUDERO, Pedro, “La Tercera Conferencia Internacional de la Alimentación. Síntesis de sus deliberaciones. Conclusiones que se desprenden”, en Boletin Oficina Sanitaria Panamericana, Washington, 1940, año 19, Na 4, pp. 330-336.

6. PERNET, Corine, "Developing Nutritional Standards 
Si bien el análisis de la situación alimentaria de la población era el objetivo central del reporte expuesto por el Dr. Escudero, sus fundamentos teóricos y metodológicos sostenían la imposibilidad de estudiar aquel problema aislado de los aspectos de las condiciones de vida asociados al trabajo o la vivienda; es decir, de aquellos "factores económicos y sociales que agitan el ambiente en el que se vive". ${ }^{7} \mathrm{La}$ investigación fue presentada entonces como una encuesta de "gran envergadura de tipo higiénico-económico" que contemplaba "a la vez todos los problemas que afectan a las familias de los trabajadores" 9 y los organizaba en tres dimensiones de pesquisa: económica, higiénica y social.

Entendemos que este relevamiento realizado entre 1936 y 1937 constituye un significativo aporte, poco estudiado hasta el momento, a la producción de información estatal sobre los problemas sociales en nuestro país. ${ }^{10}$ En este sentido, sus objetivos explícitos de integración de distintas dimensiones analíticas en el abordaje de la vida familiar, la extensa reflexión metodológica sobre los procedimientos utilizados para la recolección de los datos requeridos a las familias encuestadas, las referencias a las experiencias

and Food Policy: Latin American Reformers between the ILO, the League of Nations Health Organization, and the Pan-American Sanitary Bureau", en KOTT, Sandrine y DROUX, Joëlle, Globalizing Social Rights: The International Labor Organization and Beyond, Palgrave Mc Millan, New York, 2013 pp. 249-261.

7. ESCUDERO, Pedro, La politica..., Op. Cit., p. 64.

8. Ibídem.

9. Ibídem.

10. Aspectos de la política alimentaria impulsada por el Dr. Escudero pueden leerse en RAMACCIOTTI, Karina, La politica sanitaria del peronismo, Buenos Aires, Biblos, 2009. y las herramientas técnicas internacionales de relevamiento similar y el trabajo colaborativo e interdisciplinario de las diversas instituciones involucradas en su realización, permiten acercarse a los modos concretos en los que las técnicas de investigación social contribuyeron a la producción de diagnósticos y respuestas singulares a problemas sociales, en una coyuntura caracterizada por el fortalecimiento institucional de la intervención estatal y el creciente interés por el registro económico de las unidades domésticas. ${ }^{11}$

El objetivo declarado de la encuesta era contribuir al diseño y ejecución de políticas sociales con la expectativa de que los resultados del relevamiento constituyeran un "compás", una herramienta de orientación de la "acción futura de gobierno". ${ }^{12}$ En este sentido, el propio Instituto Nacional de la Nutrición, impulsor del estudio, se situaba en el lugar de enunciación de un consejero experto del gobierno en referencia a un nudo problemático considerado de fundamental importancia para la vida social en su conjunto: la relación entre el salario, el consumo familiar de alimentos y la salud. Los resultados publicados entrelazan el análisis de los presupuestos familiares,

11. GONZALEZ BOLLO, Hernán, La fábrica de las cifras oficiales del Estado Argentino (1869-1947), Universidad Nacional de Quilmes, Bernal, 2014, pp. 199215, GONZALEZ BOLLO, Hernán, “Ciencias Sociales y Sociografía Estatal. Tras el estudio de la familia obrera”, en Estudios Sociales, Santa Fe, Año IX, 1999, pp. 19-39; BALLENT, Anahí y GORELIK, Adrián, "País urbano o país rural: la modernización territorial y su crisis", en CATARUZZA, Alejandro (director) Nueva Historia Argentina, T. VII, Los años treinta, Sudamericana, Buenos Aires, 2002.

12. ESCUDERO, Pedro y ROTHMAN, Boris, "La vivienda en 600 familias de Obreros y Empleados de la Ciudad de Buenos Aires", en Instituto de la Nutrición, Buenos Aires, 1938, p. 132. 
con el cálculo de los valores monetarios y alimenticios mínimos requeridos para el sostenimiento de la vida (biológica y social), la descripción de la composición familiar, sus modos de obtención de recursos y la capacidad de los salarios para cubrir sus requerimientos cotidianos de alimentación, vivienda, viáticos y vestido. Se destaca en el estudio la gravitación fundamental alcanzada por la denominada "protección por la vivienda" como modo de intervención pública y privada, tanto en términos higiénicos como económicos, y por ende determinante de las condiciones de vida de la población de la Ciudad de Buenos Aires. Desde el Instituto Nacional de la Nutrición se alertaba sobre la mala alimentación de los trabajadores y las graves consecuencias morales y sociales que podrían derivar de esta situación, en términos de un llamado de atención para la acción de la política social.

Desde una perspectiva analítica atenta a los procesos de conformación de una domesticidad moderna, en y por las políticas sociales, esta encuesta nos permite comprender algunos de los modos singulares en los que se problematizó y delimitó el "hogar" en tanto unidad de análisis e intervención en pos de la "normalización" las familias objeto de intervención, atribuyéndole cualidades específicas por medio de una serie de tecnologías de registro y clasificación. ${ }^{13}$ A los efectos de este trabajo, entendemos

\section{AGUILAR, Paula Lucía, El hogar como problema y} como solución. Una mirada genealógica de la domesticidad a través de las politicas sociales. Argentina 1890-1940,

Ediciones del Centro Cultural de la Cooperación, Buenos Aires, 2014. En este trabajo se analiza, a partir de un relevamiento documental extenso, la emergencia del "hogar" como núcleo que condensa las preocupaciones morales, higiénicas y económicas sobre las condiciones de vida y trabajo de la población. El capítulo 5 "Conocer para Gobernar”, se centra en la descripción de las técnicas de registro y clasificación, aquí sólo esbozadas. que el "hogar", centro de las disputas por los diagnósticos y respuestas formulados desde las políticas sociales, no preexiste al conjunto de prácticas y saberes expertos que lo conforman y habilitan para la intervención estatal. La encuesta aquí estudiada permite observar tanto la conformación del hogar como unidad económica a través de una trama de escritura que capta sus movimientos cotidianos y los pone a disposición para la acción de gobierno, como incorporar la dimensión nutricional a la reflexión acerca de los recursos y hábitos de consumo de las familias obreras y, por lo tanto, participar de la disputa por la determinación del nivel "mínimo" de salarios adecuado para una vida saludable que permitiera la reproducción del trabajador y su familia.

En el informe que acompaña a los datos resuena la persistente inquietud por la conformación y reforma del "hogar" de la denominada "familia obrera" con características bien definidas: escindido del lugar de trabajo, higiénico, ahorrativo, bien administrado, habitado por una familia nuclear en una vivienda unifamiliar, con una clara división sexual del trabajo. Así, un conjunto de prescripciones acerca de la reforma de los hogares obreros existentes sedimenta en el registro de sus cualidades y el diagnóstico de su situación provisto por la encuesta. En suma, es posible dar cuenta a través de ellas de los rasgos de una domesticidad entendida como deseable. La noción (material y simbólica) de "hogar" emerge entonces de múltiples instancias de registro y condensa un conjunto de sentidos que buscan orientar las intervenciones estatales hacia la "normalización" de la familia obrera.

Este artículo se organiza en tres secciones. En primer lugar, se describen las características principales de la encuesta, sus objetivos 
y los criterios teórico-metodológicos del relevamiento. Luego, el análisis se detiene sobre la inteligibilidad del hogar entendida como un efecto del estudio de sus recursos y consumos e inscribe este relevamiento en una serie más amplia de estudios locales y extranjeros sobre presupuestos familiares desarrollados durante la década de 1930. Seguidamente, se examinan aquellos elementos relevados por la encuesta que permiten describir las cualidades atribuidas a lo doméstico que emergen como efecto de una cierta distribución del espacio, una división del trabajo específica y aspectos de la cultura material de los hogares relevados. Para finalizar, nos detenemos brevemente en la relación entre hogares, consumo, nutrición y salario en el marco del foro de expertos reunido en Buenos Aires que expuso la situación latinoamericana ante los entendidos internacionales en la materia.

\section{Una "investigación social"}

La realización de la encuesta estuvo a cargo de un conjunto de egresadas de la Escuela de Servicio Social del Museo Social Argentino. ${ }^{14}$ De acuerdo con el Dr. Escudero, la tarea por delante requería de personal entrenado que dominara "los problemas sociales y... [poseyera] la técnica y la disposición anímica para abordarlos". ${ }^{15}$ A ellas se sumaron algunas estudiantes de la escuela de dietistas

14. ZIMMERMAN, Eduardo, Los liberales reformistas. La cuestión social en la Argentina (1890-1916), Buenos Aires, Editorial Sudamericana, 1994. PELOSI, Hebe Carmen, El Museo Social Argentino y la Universidad del Museo Social Argentino. Historia y Proyección (1911-1978), Universidad del Museo Social Argentino, Buenos Aires, 2000.

15. ESCUDERO, Pedro y ROTHMAN, Boris, "La vivienda en...", Op. Cit., p.5.

164 y colaboradoras del Instituto Nacional de la Nutrición. La selección de quienes tendrían la responsabilidad de estar en contacto asiduo con las familias encuestadas no quedó librada al azar. El proceso de capacitación técnica y examinación rigurosa de las aspirantes constituyó uno de los principales recaudos técnicos tomados a los efectos de garantizar la confiabilidad de la información recogida. Tras aprobar las sucesivas instancias de examen eliminatorias dispuestas por el Instituto, se formuló una nueva categoría para denominar a las muchachas seleccionadas: devinieron formalmente "Investigadoras Sociales" y pasaron a depender de la Subsección de Investigación Social de la institución.

Una vez elegidas las investigadoras sociales era menester definir las familias a entrevistar. Para ello, se tomó como base una muestra relevada dentro de los límites de la ciudad de Buenos Aires. Según indica el informe fueron contactadas 2.635 familias, iniciándose encuestas en 873 de ellas. Las entrevistas completas se concretaron con un total de 600 familias, equivalentes a 3.002 personas, durante un mes completo. ${ }^{16} \mathrm{La}$ extensión mensual del registro buscaba la captación de distintas periodicidades en el pago de los salarios. Era usual que los obreros cobraran por quincena, mientras que los empleados recibían su pago mensualmente y desconocer esta situación hubiera podido afectar los resultados.

Asimismo, se tuvo en cuenta la dispersión geográfica de las familias a encuestar con el objetivo de abarcar barrios de distintas características habitacionales y ocupacionales. Se delimitaron entonces 38

16. ESCUDERO, Pedro y ROTHMAN, Boris, "La vivienda en...", Op. Cit., p.11. 
"grupos geográficos" que cumplían con esas condiciones y se llevaron a cabo algunas encuestas de tipo "experimental" para terminar de ajustar el formulario y llegar a su versión definitiva de 32 páginas. El ordenamiento de la información relevada llevó más de un año y medio y se efectuaron en total unas 26.190 visitas a las familias encuestadas. ${ }^{17}$

El contacto con las familias se realizó a través de una trama de instituciones de asistencia social, tanto laicas como religiosas, públicas y privadas que colaboraron en la investigación. ${ }^{18}$ Esta articulación institucional allanó el proceso de acercamiento a las familias potencialmente "encuestables" o sea "capaces de comprender la finalidad buscada" y que tenían "la satisfacción de tomar parte en ella prestándose pasivamente a ser estudiadas" ${ }^{19}$ Mientras que el Instituto Nacional de Nutrición ofrecía una atención integral a las familias participantes de la encuesta, las instituciones de asistencia facilitaban el acceso a las familias. Según se describe en el informe, algunas de ellas solían abrir las puertas a las investigadoras ante la expectativa de acceder a los servicios sociales y los de atención de la salud ofertados por la institución.

El anonimato de las familias encuestadas

17. Ídem, p.10.

18. Prestaron colaboración para la realización de la encuesta: la Comisión Nacional de Casas Baratas, la Administración autónoma de propiedades municipales, Asociación damas de Caridad de San Vicente de Paul, Gerencia del ferrocarril Sud, Hogares policiales secciones $1^{\mathrm{a}}$ y $17^{\mathrm{a}}$ Acción Católica Argentina y la Cooperativa el Hogar Obrero.

19. ESCUDERO, Pedro, "El estado actual de la vivienda popular en la Ciudad de Buenos Aires", ponencia presentada en Primer Congreso Panamericano de la Vivienda Popular, Buenos Aires, 1939, p. 134. eso Panamericano de la Vivienda Popular”, 1939, p. 134. era mantenido para preservar lo que el estudio denomina "pudor familiar" registrando a cada una bajo un nombre supuesto o un número. Más allá de esta precaución metodológica de distanciamiento al momento del procesamiento de la información, la relación estrecha con la familia se consideraba garantía de la "veracidad" de los datos. La mirada atenta de la investigadora social debía detectar las respuestas "poco sinceras" y hasta incluso tenía la potestad de interrumpir el curso de la indagación ante la sospecha de que la información proporcionada no fuera del todo confiable. Así, la estrategia de relevamiento utilizada constituía una práctica de caracterización económica y moral integral de los hábitos de la familia investigada:

En el cuestionario figura un rubro de observaciones y opinión personal de la investigadora social; en las primeras se anota el grado de sinceridad de lo manifestado; en la segunda se consignan las características del núcleo familiar en lo referente a los afectos y cohesión familiar, nivel de cultura, problemas económicos sociales que no surgían de los datos de la encuesta, es decir se considera a la familia como una unidad social. $^{20}$

Las visitas diarias a las familias, eran definidas como una verdadera "toma de control” de la unidad doméstica por parte de las investigadoras quienes convivieron con ellas durante un mes. En cada visita, medían $\mathrm{y}$ pesaban sus alimentos, contabilizaban sus consumos e ingresos, y rellenaban extensas planillas con información sobre la situación sanitaria y laboral de cada integrante. Las investigadoras eran a su vez supervisadas por miembros del Instituto Nacional de la

20. ESCUDERO, Pedro y ROTHMAN, Boris, "La vivienda en...", Op. Cit., p. 9. 
Nutrición en todos los pasos del estudio. Dos criterios principales se combinaban a la hora de seleccionar las familias: su situación habitacional y el rango de ingresos percibidos. Se tomó en cuenta la presencia o ausencia de "protección por la vivienda": es decir la disponibilidad de algún tipo de asistencia institucional para la adquisición o alquiler de la vivienda que ocupaban, cualquiera fuera su tipo.

La elección de la "protección por la vivienda" como criterio para clasificar las familias a encuestar se basaba en aspectos tanto económicos como higiénicos: por un lado, la importancia de los gastos en vivienda sobre el total de los ingresos del grupo familiar y, por el otro, la influencia fundamental que las condiciones de habitación tenían para la salud de la familia y de la población en su conjunto. Del total de las 600 familias finalmente encuestadas, 290 contaban con algún tipo de protección por la vivienda y 310 no contaban con protección para el pago de su alojamiento. Como veremos, esta diferencia era fundamental a la hora de calcular el porcentaje de los ingresos familiares empleados en sostener un techo y la disponibilidad de dinero para costear el resto de sus consumos.

Un segundo criterio para definir a las familias encuestables fue la delimitación de rangos de ingresos, tanto familiares como individuales. Para ello se tomaron en cuenta las familias que contaban con ingresos entre 75 y 500 pesos. Un aspecto interesante de la delimitación de los rangos es que a la hora de analizar los consumos alimenticios la cifra se combinaba además con el cálculo del ingreso per cápita en promedio, diario y mensual. La alimentación era considerada como una práctica individual, y que debía analizarse en relación a los requerimientos específicos de adultos y niños.

Se proponía discriminar rangos de ingresos en categorías por familia y por integrante y combinarlas significativamente para entender la relación entre los recursos y su distribución en relación a la composición y el tamaño de la familia. Esto permitía evaluar sus posibilidades específicas para alcanzar condiciones de vida adecuadas. Asimismo los presupuestos familiares permitían captar la participación de las mujeres y los niños en el mercado de trabajo y su contribución a los recursos del hogar.

El estudio se enmarcaba teóricamente en lo que se daba en llamar "profilaxis no específica", es decir, aquella que tenía por objetivo no sólo las medidas necesarias para el combate de los agentes productores de enfermedades ya conocidas, sino el cuidado de las condiciones sociales que limitaban las capacidades y por ende "acortan la vida" de los trabajadores considerados sanos.

El cuidado permanente del hombre sano, en todos los períodos evolutivos de su vida, constituye el objeto de la profilaxis no específica; para ello es indispensable gobernar su alimentación y su trabajo cuidar de la vivienda y del vestido. Como el hombre es una unidad in divisible del cuerpo y del espíritu, ambos deben ser cuidados con la misma atención. ${ }^{21}$

Se trataba de una aproximación médica que incorporaba la dimensión social de la salud de la población. El Instituto Nacional de la Nutrición contaba ya con antecedentes en la sistematización de la información sanitaria de quienes se acercaban a solicitar sus servicios en

21. ESCUDERO, Pedro, "La alimentación de la Familia...”, Op. Cit., p. 4. 
los consultorios externos de la institución sin presentar (al menos en apariencia) problemas de salud, y reivindicaba la idea de una medicina del "hombre sano" que "vive de su trabajo" y en la que la alimentación y su estudio jugaban un rol fundamental. ${ }^{22}$

A continuación, nos centraremos en la información proporcionada por las secciones "Económica" e "Higiénica" de la encuesta, y, en menor medida, de aquella identificada como "Social", donde se presenta la composición de las familias encuestadas. La disposición de la información bajo la técnica del presupuesto familiar nos permite poner en serie este relevamiento con otras experiencias de construcción de conocimiento estatal sobre las familias en curso por esos mismos años.

\section{Presupuestos familiares, necesidades y recursos}

Conocer la composición de los recursos de los que disponen las familias y los efectos que su administración conlleva en la determinación de las condiciones de vida constituye un objetivo básico de la construcción del conocimiento necesario para la regulación de la fuerza de trabajo. En el plano internacional los estudios que adoptan como unidad de observación los presupuestos familiares tienen una larga tradición ${ }^{23}$. Sin embargo, éstos cobran un

22. Esta posición fue ampliada en el trabajo

ESCUDERO, Pedro, "Los requerimientos del hombre sano y normal y Las encuestas de alimentación, ponencia presentada en XI Conferencia Sanitaria Panamericana, Rio de Janeiro, 1942, Instituto Nacional de la Nutrición, Ministerio del Interior, 1943.

23. LAZARSFELD, Paul, "Afterword. Toward a History of Sociography”, en LAZARSFELD, Paul, JAHODA, Marie y ZEIZEL, Hans Marienthal. The Sociograpphy of renovado interés a mediados de la década del 1920 a partir del impulso brindado por organizaciones internacionales como la OIT, entre otras, a la realización investigaciones comparativas a nivel internacional sobre el poder de compra de los salarios. El interés por la relación entre salario real y la composición familiar se reaviva tras la crisis económica de 1930, multiplicándose las experiencias de cuantificación. ${ }^{24} \mathrm{La}$ disputa política $\mathrm{y}$ metodológica por la estandarización de los modos de relevar la información sobre los hogares va conformando paulatinamente un consenso internacional sobre la necesidad de realización de encuestas que registraran en detalle los presupuestos familiares y el costo de vida en cada país. ${ }^{25}$

Entre 1935 y 1937, la relación entre salario y nutrición se problematiza a partir del impulso brindado por la Oficina Sanitaria de la Liga de las Naciones a la ejecución de encuestas nacionales sobre la alimentación. ${ }^{26}$ Si bien la efectiva realización de las encuestas contó con la colaboración de la Organización Internacional del Trabajo (OIT), algunos investigadores señalan la existencia de matices en la orientación de los relevamientos

an unemployed community, Aldine Atherton Inc, Chicago, 1971, p. 99-115; JOHNSON, David, ROGERS, John y TAN, Lucilla, "A century of family budgets in the United States", en Monthly Labor Review, May, 2001.

24. WILLIAMS, Faith y ZIMMERMAN Carle, Studies of family living in the United States and other countries: An analysis of material and method United States, Dept. of Agriculture, Washinnçgton D. C., 1936.

\section{INTERNATIONAL LABOUR OFFICE}

STUDIES AND REPORTS, "Methods Of Conducting family Budget Enquiries”, Geneva, Londres, 1926.

26. CAMPBELL, Janet, “The Nutrition Report”, en International Affairs (Royal institute of International Affairs 1931-1939), 1938, V. XVII, No. 2, pp. 251-253. 
conjuntos. $^{27}$ Mientras que los objetivos de la OIT estaban más orientados hacia los cálculos comparativos de los salarios reales y su poder de compra, la Oficina Sanitaria de la Liga de las Naciones, por su parte, priorizaba la mirada médica sobre la nutrición que implicaba mayor complejidad en tanto suponía el relevamiento de los nutrientes alimenticios y de los hábitos familiares. ${ }^{28} \mathrm{La}$ necesidad del estudio de las consecuencias sociales de la crisis de 1930 generó espacios de trabajo conjunto entre ambas organizaciones y la problematización de la nutrición cobró relevancia internacional en esa coyuntura. La realización de la Tercera Conferencia Internacional sobre la alimentación en Buenos Aires durante 1939, sumó al debate la posición de los expertos latinoamericanos quienes solicitaban a ambas instituciones internacionales el apoyo técnico y económico para la realización de estudios nacionales que permitieran dar cuenta de las características específicas que adoptaba el problema nutricional en cada territorio.

A nivel local tras las primeras incursiones del Departamento Nacional del Trabajo en la realización de encuestas sobre los gastos de las familias en la Ciudad de Buenos Aires se desarrollaron en las primeras décadas del siglo XX. ${ }^{29}$ En 1933, se realiza una encuesta sobre

27. WEINDLING, Paul, "Social medicine at the League of Nations Health Organization and the International Labor Office compared", en WEINDLING, Paul (editor) International Health Organizations and Movements (1918-1939), Cambridge University Press, Cambridge, 1995, pp. 134-149.

28. BIGWOOD, Édouard Jean, Guiding principles for Studies on the Nutrition of Populations, League of Nations Health organization, Technical commission on Nutrition, Geneva, Columbia University Press, New York, 1939. Estas instrucciones fueron discutidas en el marco de la Tercera Conferencia Internacional sobre la alimentación.

29. GONZALEZ BOLLO, Hernán, "La cuestión 168 los consumos de trabajadores ${ }^{30}$, que daría lugar a la definición de una canasta más amplia de productos cuyos precios serían relevados periódicamente.Uninforme de su actualización a los precios vigentes en 1935 fue publicado bajo el título de "Condiciones de vida de la familia obrera" ${ }^{31}$ El relevamiento enumeraba los productos y cantidades consumidos por las familias a partir de su clasificación en rangos de ingreso y la construcción de un índice que representaba la capacidad de consumo de cada uno de sus miembros. El informe centraba su atención en los salarios más bajos (120 pesos) y buscaba captar su sensibilidad a las oscilaciones de los productos de "primera necesidad". Esta grilla de observación brindaba la posibilidad de categorizar cada una de las familias encuestadas a partir de la relación entre sus ingresos y la capacidad para cubrir estos artículos. Así se establecía la situación de presupuestos/familias en "déficit" o "superávit".

La recolección y presentación de la información bajo la forma de presupuesto familiar permitía visibilizar la relación entre ingresos y consumos, habilitando la formulación de categorías para agrupar a

obrera en números: La estadística socio-laboral argentina y su impacto en la política y la sociedad, 1895-1943", en OTERO, Hernán (director) El Mosaico Argentino. Modelos y representaciones del espacio y la población, Siglos XIX y XX, Siglo XXI Editores, Buenos Aires, 2004.

30. REPÚBLICA ARGENTINA, Departamento Nacional del Trabajo, "Instrucciones para realizar la investigación del costo de la vida de la población obrera de la Capital Federal",1933.

31. MARSHALL, Adriana, "La composición del consumo de los obreros industriales de Buenos Aires, 1930-1980", en Desarrollo Económico, V. XXI, No 83, (Oct.-Dec 1981); REPÚBLICA ARGENTINA, Departamento Nacional del Trabajo - División de Estadística, "Condiciones de Vida de la Familia Obrera", Buenos Aires, 1937. 
los hogares que se encontraban en situación similar. Las mediciones del Departamento Nacional del Trabajo compartían con la encuesta realizada por el Instituto Nacional de la Nutrición tres aspectos, a saber: el establecimiento de rangos de salarios para delimitar las familias trabajadoras, un registro de aquellos productos y servicios que era posible comprar con los importes de los salarios considerados, y la relación entre composición familiar, necesidades y recursos.

Sin embargo, la encuesta del Instituto Nacional de la Nutrición, incorpora a la información producida sobre los costos de vida de la familia obrera por el Departamento Nacional de Trabajo, el desafío de captar la nutrición de la población a través de los gastos reales destinados a su alimentación cotidiana y su relación con los requerimientos calóricos necesarios para conservar la salud. A partir de estas pautas, las Investigadoras Sociales debían registrar los precios efectivamente pagados por las familias y no los precios del mercado tomados como referencia para cuantificar una lista o canasta de productos previamente formulada. Esto permitía captar los valores monetarios necesarios para adquirir los alimentos entendiendo que sufrían variaciones no sólo estacionales sino geográficas.

En este punto, el estudio del Instituto Nacional de la Nutrición se acerca más a una observación cualitativa, capilar, de la cotidianeidad de las familias de distintos ingresos y hábitos alimenticios, que a un relevamiento cuantitativo del poder de compra del salario. A los efectos de los objetivos del Instituto no era suficiente con estudiar de modo "aislado" y "aritmético" el presupuesto de las familias sino que interesaba conocer "cómo cada familia emplea sus recursos" en relación con la salud. Así, si una familia tenía superávit porque "vive miserablemente con desgaste anormal de la vida", tal superávit nada decía de su condición. ${ }^{32}$ El horizonte era poder reorientar las prácticas cotidianas de la familia en pos de obtener una mejor utilización de los recursos y así promover una alimentación adecuada.

Este afán por captar el consumo real de las familias supuso estudiar y reproducir las mismas condiciones materiales en las que éstas elaboraban y consumían sus alimentos. El objetivo declarado era el de mejorar la eficiencia de los modos de adquirir (cantidades y variedad) los alimentos, pero también de prepararlos. En este sentido, la investigación se distancia explícitamente de los cálculos de costo de vida del Departamento Nacional del Trabajo cuando afirma que "algunos se contentan con conocer los precios oficiales, calcular aproximadamente el consumo de alimentos de la familia y deducir" ${ }^{33}$, sin considerar las diferencias notables de cada grupo familiar en las prácticas de adquisición y preparación.

Desde la perspectiva del Instituto, el costo real de la alimentación de la familia estaba compuesto por dos valores complementarios: a) el precio que el consumidor pagaba por los alimentos en las ferias, los mercados y almacenes y b) el precio del combustible utilizado en la preparación de los alimentos. ${ }^{34}$ La clave del estudio era entonces "comprar

32.ESCUDERO, Pedro, La Política Nacional de la Alimentación en la República Argentina, Ministerio de Relaciones Exteriores y Culto, Buenos Aires, Instituto Nacional de la Nutrición, 1939, p. 94.

33. ESCUDERO, Pedro, "La alimentación de la Familia...”, Op. Cit., p. 28.

34. Ibídem. 
en los mismos lugares donde todos compran, utilizar el combustible que la mayoría emplea, preparar los alimentos con los mismos utensilios y equipo de cocina" con que contaba la familia". ${ }^{35} \mathrm{El}$ informe aclara que no incluye en su cálculo del costo "real" de la alimentación el tiempo empleado por quien realiza la tarea de prepararla (la esposa) dado que consideraba su misión específica "cuidar todos los menesteres del hogar"

La perspectiva de la profilaxis específica que anima el estudio centra el interés en la nutrición en tanto proceso biológico/fisiológico con consecuencias sociales. Esta matriz médica se hace presente al momento de definir y poner en relación las necesidades y los recursos de las familias. Éstas son definidas como "exigencias mayores de la vida vegetativa: alimentación, trabajo, vivienda y vestido". ${ }^{36}$ En este sentido, el informe afirmaba que hasta ese momento había predominado un "concepto económico" al estudiar las relaciones sociales, sintetizable en la frase "capital y trabajo" que no se había modificado demasiado con la incorporación de demandas de corte higienista en pos del trabajador y que desde la perspectiva del Instituto Nacional de la Nutrición, debía primar un criterio de análisis donde "la formación, conservación y rendimiento de la vida" fuera considerado como el criterio básico de análisis; y por lo tanto, la faz económica se ajustara a la faz biológica, estableciendo los mínimos necesarios para vivir de su trabajo. ${ }^{37}$

35. Ibídem.

36. ESCUDERO, Pedro, “E1 Estado actual de...", Op.

Cit. p. 131.

37. Ídem, p. 124.

170
Cada hombre necesita un mínimum de alimentación para mantener su integridad fisiológica y el rendimiento de su trabajo; cada familia necesita un mínimum de exigencias en la vivienda y en el vestido para mantener el decoro y la moral; un mínimum de exigencias espirituales, recreación, cultura, religión, para mantener la dignidad humana. Cada hombre debe subvenir con su trabajo a sus necesidades y a las de la familia que ha constituido, pero el rendimiento del esfuerzo está supeditado al estado físico y a la cultura. ${ }^{38}$

Estos "mínimos", debían cubrirse con los recursos expresados en el presupuesto familiar, entendiendo por "recursos" todo aquello que permitiera cubrirlas, así fuera dinero proveniente del salario u otros ingresos: "el valor en efectivo obtenido por trabajo, renta, donación y jubilación; el valor de la propiedad traducido por su renta probable, el valor recibido en especies, en ayuda alimenticia, en vestidos, en movilidad". ${ }^{39}$ Una vez procesada la información recogida por las investigadoras, los resultados daban cuenta de la siguiente distribución de los recursos recibidos por las familias: en dinero efectivo 79\%, en especie el $19 \%$, en vivienda el $1 \%$ y en medios de locomoción el 1\%. La jerarquización del peso de cada erogación en el presupuesto familiar demostraba el rol fundamental de la vivienda en los gastos familiares. Al ordenar la distribución de los gastos de los hogares por rubros encontramos en primer lugar la vivienda e iluminación, luego la alimentación y el combustible, en tercer lugar los viáticos, y en cuarto lugar el vestido.

38. Ibídem.

39. Ibídem. 
Según el Dr. Escudero, el análisis de los presupuestos brindaba además información que permitía "formar juicio de las dificultades del interior familiar" ${ }^{40}$, ya que dependía del monto de los recursos con que contaba cada presupuesto familiar la existencia de una determinada división del trabajo entre varones y mujeres: "Cuando sólo el esposo soporta todo el peso del presupuesto familiar, la esposa puede dedicarse al cuidado y vigilancia de los hijos y del esposo". ${ }^{41}$ Las formas de la organización familiar se presentaban estrechamente relacionadas con los recursos disponibles y la contribución de cada miembro al presupuesto total de la familia.

En este sentido, los resultados de la encuesta mostraban que el $96 \%$ de los esposos contribuían a cubrir la parte mayor del presupuesto familiar. En el $84 \%$ de los casos lo hacían a través del trabajo, el $10,05 \%$ por jubilación o renta y el $1,4 \%$ por otros recursos. ${ }^{42}$ En más del 50\% de los casos, el trabajo del esposo cubría el presupuesto familiar en un 90 a $100 \%$ del total de los gastos. Es significativo que el estudio registrara también en la cuantificación de los recursos el aporte de las "esposas". Así, sostiene que de las 584 esposas encuestadas, el $50 \%$ no aportaba recursos al hogar y el 33\% lo hacía regularmente. De las esposas que aportaban recursos, el $45 \%$ lo hacía en dinero "obtenido como remuneración del trabajo", el 17\% en dinero por jubilación, pensión o renta y el resto por otros conceptos no especificados.

Sin embargo, el estudio planteaba que para poder valuar el costo de la vida de las personas

40. Ídem, p. 158.

41. Ídem, p.159-160.

42. Ídem, p.160. estudiadas, debía tenerse en cuenta el salario diario individual, cifra que permitía establecer la relación entre cada uno de los integrantes de la familia y los recursos totales. Para ello, era preciso conocer la composición familiar. La investigación del Instituto Nacional de la Nutrición consideraba una familia "tipo" de 5 personas, integrada en términos teóricos por los padres y tres hijos de 5, 10 y 14 años. El "presupuesto límite" de esta familia, era el equivalente al salario mínimo pagado por la Municipalidad de la Capital, que ascendía a 150 pesos. Se consideraba que por debajo de este salario "la vida se hace difícil en el ambiente de Buenos Aires y por lo tanto es la mínima retribución que es dable aceptar como viable". ${ }^{43}$ Ahora bien, aceptar este monto como base de los cálculos y de las apreciaciones económicas y sociales del estudio no significaba aceptarlo en términos normativos como una retribución conveniente para ese tipo de familia. Las familias cuyos ingresos no excedían de un peso diario por integrante, tenían serias dificultades.

Al comparar las familias que poseían "presupuestos holgados" con aquellas de "presupuestos insuficientes", se demostraba que la reducción de los recursos disponibles les obligaba a una alimentación incompleta, al uso de una vivienda reducida y el acortamiento del descanso. De acuerdo con el informe presentado por el Dr. Escudero ante la Tercera Conferencia Internacional de la Alimentación esta situación tenía graves consecuencias ya que la mortalidad infantil aumentaba en los hogares que no accedían a los mínimos necesarios.

La exposición de la información relevada

43. ESCUDERO, Pedro, "La Tercera Conferencia...", Op. Cit. p. 332. 
bajo la forma del presupuesto familiar no sólo permite un registro de los recursos y erogaciones monetarias de los hogares, sino también el diagnóstico de sus dinámicas internas y su clasificación. Así la descripción de los equilibrios y desequilibrios económicos que afrontan las familias y a partir de los cuales se las agrupa, permiten observar en el conjunto de la población estudiada el establecimiento del mínimum necesario para la reproducción de la vida, según su estado físico y cultura. Nutrición y economía se combinan en la definición de las necesidades y su satisfacción mediada por el salario, administrado por el ama de casa.

\section{Domesticidad e intervención}

La hora de la comida es la hora del hogar. ${ }^{44}$

El relevamiento del Instituto Nacional de la Nutrición permite observar la delimitación de un conjunto de cualidades como propias del hogar que emerge en tanto unidad de observación e intervención a través de la información registrada y categorías en las que ésta se organiza. Los resultados de la encuesta brindan precisiones sobre la distribución espacial del hogar en tanto "medio material donde se desarrolla la vida de la familia", la división sexual del trabajo que en él se desarrolla y el trazado de las fronteras entre trabajo y hogar. Su detallado registro permite además observar algunas huellas de la cultura material de las familias encuestadas.

Tal como se mencionara más arriba, la vivienda ocupa un lugar clave a la hora de considerar las condiciones de vida de las

44. Ibídem.

172 familias. Los resultados de la encuesta muestran que el número de habitaciones disponibles en cada unidad doméstica estaba directamente relacionado al precio de la vivienda. Así de las 300 familias sin "protección por la vivienda" encuestadas, el 45,5\% ocupan una sola pieza ${ }^{45}$, dos habitaciones en un $38,27 \%$ y tres y más habitaciones un 16,20\% del total. La relación se invierte al considerar aquellas familias que contaban con algún tipo de protección que abaratara el acceso a la vivienda. De este grupo, sólo el $1,93 \%$ de las familias con protección habitaba en una sola pieza, el 29,35\% en dos, y el 68,38\% en viviendas de tres habitaciones o más. ${ }^{46}$ De las 600 familias participantes en el estudio, el 55,5\% habitaba en viviendas individuales y el $44,5 \%$ en viviendas colectivas. De las 267 familias que conformaron este último grupo el 52,43\% ocupaban departamentos, el 25\% inquilinatos y el 22,4\% conventillos. Éstos prevalecían en las zonas céntricas mientras que, de acuerdo al informe, el inquilinato era propio de los suburbios.

Las casas construidas o amparadas por instituciones de protección social (laica o religiosa) eran más amplias y prestaban especial atención a la distribución del espacio y la división los integrantes de la familia por edad y por sexo, de modo que es razonable que predominaran en el relevamiento las viviendas más amplias en las familias con alguna forma

45. Cabe destacar que la problematización del hacinamiento y de la distribución espacial de las familias en términos que "viven en una sola pieza" es un tópico reiterado en el debate de las condiciones de vida y trabajo de la población por esos años. Por razones higiénicas y morales, el hacinamiento es fuertemente criticado y rechazado desde todos los sectores. Véase AGUILAR, Paula, "El hogar como...", Op. Cit., pp.109-153.

46. ESCUDERO, Pedro “El Estado actual de...", Op. Cit., pp. 10-12. 
de protección. La preferencia normativa de la casa individual por sobre la colectiva se hace explícita en el análisis de los datos de la encuesta. ${ }^{47}$

Además de tener en cuenta el número de habitaciones, el informe construye un interesante índice para medir el hacinamiento que asocia la cantidad de camas y de habitantes de la vivienda que las utilizan. Esta relación, llamada densidad "plaza/cama", indicaba la cantidad de personas que ocupaban un mismo dormitorio y la existencia de camas suficientes para su descanso. Basado en este cálculo, un coeficiente cercano a uno equivalía a la ocupación de una persona por lecho, mientras que si superaba esta cantidad, se suponía que una misma cama era utilizada por más de una persona y por tanto, permitía captar el hacinamiento. A partir de los datos recogidos, el estudio estableció que el $50 \%$ de los casos de familias sin protección por la vivienda el coeficiente era "superior a la unidad y por lo tanto anormal". ${ }^{48}$ Se advierte la agravada densidad de población de los dormitorios como un signo de alerta en términos higiénicos y morales.

Es posible además captar huellas de la cultura material ${ }^{49}$ de las familias encuestadas a

47. Esta posición era compartida por sectores conservadores y católicos, quienes por esos años presentaron propuestas al respecto en el Congreso Panamericano de la Vivienda Popular, realizado en 1939.

48. ESCUDERO, Pedro y ROTHMAN, Boris, "La vivienda en...", Op. Cit., p. 31.

49. PESEZ, Jean Marie, "Historia de la cultura material”, en DE LAS TRAVIESAS MORENO, Luis y ALONSO GONZALEZ, Gladys (editores) La Historia y el oficio de historiador: Colectivo de autores francés, Editorial de Ciencias Sociales, La Habana, 1996; SANCHEZ, María Inés, El espacio doméstico en Buenos Aires (1872-1935): Concepciones, modelos e imaginarios, través de las menciones que el estudio realiza acerca de los utensilios y artefactos disponibles en los hogares. Esta información se propone fundamental para poder reproducir en las cocinas del Instituto Nacional de la Nutrición las prácticas de cocción de los alimentos bajo las mismas condiciones que éstas se efectuaban en los hogares. Se esperaba que el relevamiento permitiera a las investigadoras adecuar la formación doméstica propuesta para las familias encuestadas y otras que así lo solicitaran. Dado que la encuesta estaba orientada hacia la relación entre alimentación y salud, el relevamiento prestó especial atención a la cocina como espacio físico necesario para la preparación de los alimentos, pero también a la cocina en tanto "aparato" utilizado para cocinar. El estudio evaluaba positivamente que el $98 \%$ de las 600 familias encuestadas tuvieran "local cocina" propio, mientras que el 0,66\% poseía una cocina en común con otras familias, y que sólo un $1,16 \%$ de las familias no tuvieran cocina y prepararan sus alimentos en el patio o en el dormitorio.

Respecto de la cocina en tanto "artefacto", a través de la información recogida es posible también saber que el $95 \%$ de las familias contaban con equipos de cocina de "primer orden", que en el 65,83\% de los casos la cocina contaba con dos hornallas, el 22,16\% disponía de tres o más hornallas, y sólo en el $6 \%$ de los casos se contaba con una sola. Este dato era considerado importante en términos de las prácticas culinarias disponibles para las familias, ya que la existencia de más de una hornalla en la vivienda permitía la cocción de más de un alimento a la vez y, por tanto, suponía la posibilidad de una alimentación más compleja y variada. Respecto del

Librería Concentra, Buenos Aires, 2010. 
combustible doméstico, la encuesta planteaba que la mayoría de las familias utilizaba carbón de leña o kerosene y gas, en menor medida.

Junto a las tareas de adquisición y la preparación de los alimentos, la encuesta aportaba elementos para analizar la división del trabajo dentro y fuera del hogar. En ella, se asociaba la presencia femenina en el hogar a la cohesión familiar y a la posibilidad de una alimentación adecuada. De acuerdo con el Instituto de la Nutrición, los datos acerca de la dedicación "habitual de la madre" permitían reconocer el "grado de estructuración de la familia" ${ }^{50}$. Así, una madre podía estar "en el hogar, cuidando de la familia" o "fuera de él, trabajando en busca de recursos". ${ }^{51}$ Esta situación variaba de acuerdo con los recursos totales del grupo familiar. En las familias que contaban protección por la vivienda, el $44,52 \%$ de las mujeres estaban "dedicadas exclusivamente al cuidado del hogar" ${ }^{52}$ y en aquellas que no contaban con protección sólo un 32,70\% de las mujeres permanecían en él. Un segundo grupo estaba conformado por las que realizaban algún tipo de trabajo remunerado en el hogar. En ese caso quienes "cuidan del hogar y tienen trabajo lucrativo en él" 53 asciende al 2,73\% de las mujeres en las casas con protección y al 8,38\% en aquellas sin protección. En total, el informe destacaba que la ocupación de la esposa constituía en más del $77 \%$ de las familias encuestadas "el cuidado del hogar" mientras que el resto se repartía entre el trabajo lucrativo en el hogar y el trabajo fuera de éste. Para concluir señalaba este "alto por

50. ESCUDERO, Pedro, "La alimentación de la

Familia...”, Op. Cit., p. 12.

51. Ibídem.

52. Ídem, p. 13.

53. Ibídem.

174 ciento de madres dedicadas al hogar es una cifra tranquilizadora". ${ }^{54}$

Sugiere la encuesta que estas mujeres dedicadas al hogar eran las que realizaban primordialmente las compras destinadas a la alimentación. Este gasto, según la encuesta, representaba la inversión mayor de las familias con presupuestos modestos, que invertían el $50 \%$ de sus entradas en alimentación. Sin embargo, advierten que el mayor gasto familiar se realizaba sin ningún "criterio económico" que permitiera conocer los valores nutritivos de los alimentos a adquirir y poder elegir en consecuencia. Esta falta de formación para la correcta selección de los alimentos más adecuados para la nutrición familiar daba paso a la compra de productos "de acuerdo al gusto o capricho de la persona que hace las compras". Así, la dueña de casa, aunque animada de la mejor buena voluntad podía por desconocimiento no comprar de modo adecuado..$^{55}$

E1 peligro mayor: La ignorancia. Lo angustioso no es sólo el precio de la alimentación, siempre elevado, sino esta otra situación: en general se invierte mal el poco dinero disponible para alimentarse, y esto no sólo ocurre entre nosotros; se ha demostrado en todas partes donde el tema ha sido estudiado. ${ }^{56}$

54. ESCUDERO, Pedro, "La Alimentación de la familia en Buenos Aires", Ministerio de Relaciones Exteriores y Culto, Instituto Nacional de la Nutrición, Buenos Aires, 1939, p. 12.

55. Afirmaciones similares pueden leerse en BUNGE, Alejandro, "Formación del sentido económico de la Mujer", en La economía Argentina, La conciencia Nacional y el problema económico, V. 1, Agencia General de Librerías y Publicaciones, Buenos Aires, 1928.

56. ESCUDERO, Pedro, "La Alimentación de la familia...”, Op. Cit., p. 106. 
En virtud de este diagnóstico y de su orientación más general hacia la educación nutricional de la población, el Instituto Nacional de la Nutrición tomó una serie de medidas de educación popular respecto del consumo y preparación de alimentos. En 1936, por ejemplo, según consigna su boletín, se iniciaron demostraciones de cocina en aquellas fábricas que empleaban mujeres, a cargo de las alumnas de la Escuela Municipal de Dietistas, en las que se daba una explicación "breve y sencilla" de los platos a elaborar y luego se hacía el análisis económico de la comida preparada descripta como un "menú de carácter popular". También disponían de la opción de enviar, por pedido de las casas colectivas, una dietista a dar explicaciones simples de los platos más comunes. Si bien habían preparado un camión con una cocina ambulante para esta tarea, la actividad no tenía mayor aceptación entre los posibles destinatarios. Para estas iniciativas de instrucción, no se empleaban más utensilios que los que poseían las casas más humildes: "cacerolas, sartenes, ollas, un hervidor de leche y un brasero o calentador a kerosene". ${ }^{57}$

A la hora de la intervención, el desafío de la política social de asistencia alimentaria tal como era propuesta por el Instituto Nacional de la Nutrición, era no incidir en los lazos familiares. Si bien el Instituto tenía larga tradición en calcular y confeccionar recetarios para comedores de grandes instituciones escolares, militares y hospitales, consideraba que la asistencia familiar debía proteger el hogar y la comensalidad común.

La hora de la comida es la hora del hogar, el motivo de reunión de la familia, de su integración espiritual. La ayuda alimenticia no de be desintegrarla, ni ser parcial con

57. Ídem, p. 15. su litación. Cada hogar debe bastarse a sí mismo, debe ayudarse por todos los medios a equilibrar su presupuesto, respetando la unidad moral y material de la familia, fundamento de la sociedad. ${ }^{58}$

El consumo de alimentos y su preparación en el seno del hogar constituía el conjunto de prácticas que mediaba entre los recursos provenientes del salario y la nutrición de los trabajadores. Si bien el monto del presupuesto disponible para cada familia definía los alimentos plausibles de ser adquiridos, el sólo aumento de los recursos no garantizaba una alimentación correcta en términos nutricionales. Es decir, la capacidad de consumo del salario no era asimilable a una buena alimentación.

A lo largo de la Tercera Conferencia Internacional sobre la Alimentación desarrollada en Buenos Aires, con la que iniciamos este artículo, esta relación entre alimentación y salarios fue un punto importante en discusión en relación con las posibles políticas a implementar. De acuerdo con la posición de la delegada de los Estados Unidos, la experta en Economía Doméstica Mrs. Stiebeling, era posible adecuar los regímenes alimenticios de la política de alimentación a la magnitud del salario. ${ }^{59}$ Sin embargo, el Dr. Escudero, representante argentino en la conferencia consideraba que, desde el punto de vista biológico, esta adaptación constituía un error.

58. ESCUDERO, Pedro, "La Tercera Conferencia...", Op. Cit., p. 333.

59. STIEBELING, Hazel (1896-1989). Dietista e investigadora norteamericana pionera en el estudio de la alimentación y la Economía Doméstica en los EEUU. 
El aumento del presupuesto familiar hasta la holgura, no asegura una alimentación correcta de la familia; que los prejuicios y la ignorancia son causa de gran desviación de la normal alimentación. No puede darse como solución, única y aislada, del problema de la alimentación de la familia obrera la elevación del salario. ${ }^{60}$

Desde la perspectiva del Instituto Nacional de la Nutrición, la alimentación debía garantizar "lo justo para vivir, conservando la salud, asegurando el rendimiento del trabajo y la normal perpetuación de la especie" ${ }^{61}$ De acuerdo con sus cálculos, cuando el $60 \%$ de un salario dejaba de cubrir estas exigencias, ya no cumplía con su "finalidad mínima". ${ }^{2} \mathrm{La}$ posibilidad de supeditar el régimen alimenticio a la remuneración sin más, era solo plausible de ser considerada en tiempos de excepción:

En condiciones de emergencia, guerras, crisis sociales, etc., puede haber una reducción transitoria del régimen alimenticio; en este caso deberá hacerse en forma que no se comprometa la salud o que este hecho se retarde. Esta reducción no puede quedar librada al acaso o a la improvisación, debe ser aconsejada por técnicos. ${ }^{63}$

Las conclusiones de la conferencia indicaban la imposibilidad material de cubrir el presupuesto de alimentación cuando las entradas no pasaban de un "límite crítico". ${ }^{64} \mathrm{En}$

60. ESCUDERO, Pedro, "La Tercera Conferencia...", Op. Cit., p. 332

61. ESCUDERO, Pedro, "La Alimentación de la familia...”, Op. Cit., p. 35.

62. Ibídem.

63. ESCUDERO, Pedro, "La Tercera Conferencia...", Op. Cit., p. 331.

64. ESCUDERO, Pedro, "La tercera Conferencia Internacional sobre la Alimentación - Síntesis de este contexto, los problemas de nutrición de la población, entendida a partir del diagnóstico de la "subalimentación" eran definidos como una tragedia que afectaba sin excepción a todos los países de América Latina donde una proporción importante de la población no alcanzaba a "comer lo mínimo que debe exigirse, para conservar la vida y permitir un rendimiento normal del trabajo humano". ${ }^{65}$ Lo que está en juego en estas intervenciones es el debate por el establecimiento de salarios mínimos y los criterios a los que debiera responder su determinación. ¿Debían establecerse mínimos con énfasis en los requerimientos nutricionales necesarios para conservar la salud o en la adaptación de éstos al nivel de retribuciones existente? Este interrogante acompaña hasta hoy los debates acerca de la definición de canastas "básicas" y salarios "mínimos".

Es interesante destacar, sin embargo, que tanto Mrs. Stiebeling como el Dr. Escudero coincidían en la necesidad de una consideración integral de la situación alimenticia y en la importancia de la educación en economía doméstica que permita garantizar la buena administración del salario y el consumo de una cantidad y calidad adecuada de alimentos. En los términos propuestos por el propio Escudero a la hora de listar propuestas de política social "una campaña educacional que capacite para el manejo del capital-alimento" y la "ayuda alimenticia o económica cuando los recursos fueran reducidos". A través de la discusión sobre nutrición es posible dar

sus deliberaciones, Conclusiones que se desprenden", Ministerio de Relaciones Exteriores y Culto, Instituto Nacional de la Nutrición, Folleto No 4, Buenos Aires, 1939 , p. 3.

65. Idem, p. 4. 
cuenta de un momento clave del debate sobre la formulación de mínimos salariales y su relación con los mínimos necesarios para el sostenimiento de la vida biológica y social. La información relevada sobre los hogares asocia las formas deseables de organización familiar a la disposición de un espacio físico adecuado para la habitación, la tenencia de artefactos e instalaciones dedicadas a la preparación de los alimentos y la presencia de las mujeres en el ámbito doméstico. Estos tópicos señalados por la información son claves para comprender la consideración del "hogar" como espacio de intervención de la política social y su articulación con la administración del salario.

\section{Palabras Finales}

El estudio exhaustivo de esta experiencia de producción estatal de información revela los modos en que el conocimiento científico, los saberes expertos acerca de lo social, van dando forma a través de sus categorías a las posibles intervenciones sociales del Estado. La encuesta realizada por el Instituto Nacional de la Nutrición, hace inteligibles, a través de sus cuestionarios y tabulaciones, los hogares de las familias obreras, detallando sus protecciones, recursos y consumos, en suma, registrando aquellas informaciones consideradas como pertinentes para sostener una mirada integral de la cuestión nutricional en tanto problema social. Sus resultados dan cuenta de familias cuyas condiciones de vida están ligadas de modo indisociable a los niveles salariales y a la propiedad o no de una vivienda, que en su mayoría dependen del ingreso principal aportado por el varón y cuya situación habitacional dista de ser adecuada a los criterios básicos de la higiene.
Se destaca en sus páginas la permanencia de las mujeres en el ámbito doméstico como un factor positivo para la administración doméstica. Si bien la gran mayoría de los hogares estudiados cuentan con medios suficientes para la adquisición de alimentos y un espacio específico para su preparación, esto no garantiza la correcta nutrición de las familias. Se espera que la educación de las encargadas de realizar las compras, revierta en parte esta situación. Asimismo, se describe una intervención minuciosa del estado sobre el conjunto de las familias encuestadas y la profesionalización de un conjunto de saberes expertos (trabajadoras sociales, expertos en nutrición) que se complementan en sus tareas de relevamiento y análisis.

El modo en que la relación entre hogares, alimentación y salarios es problematizada como objeto de políticas sociales específicas, autoriza una entrada a la reflexión sobre el consumo que lo ubica en un lugar de intersección entre la vida económica cotidiana de los hogares y la regulación estatal. El consumo es el conjunto de prácticas que media entre el salario y la alimentación. En su correcta orientación está en juego el gobierno del "capital-alimento" y por ende, la salud de la población. A través de esta problematización es posible observar la delimitación de los hogares asalariados urbanos, donde la mediación monetaria es clave para la supervivencia

La encuesta del Instituto Nacional de la Nutrición, amplia la reflexión sobre el conjunto de elementos que debiera cubrir el salario. Así, considera que se requiere un mínimum que comprende la alimentación, el vestido, y la vivienda, pero también la recreación o la cultura dentro de aquellos factores necesarios para mantener la "dignidad humana". Más 
allá del cálculo de la relación entre recursos y consumos "aritméticos" presentada por los cálculos del costo de vida, el interrogante por la salud y la nutrición profundiza el estudio de las condiciones de vida al tiempo que contribuye a la discusión sobre los criterios para el establecimiento de mínimos salariales y el poder real de compra de los trabajadores.

La captación de las prácticas de consumo y administración de los recursos in situ, solo fue posible a partir de las técnicas de la visita cotidiana por parte de las "investigadoras sociales" entrenadas para tal fin. El registro de los presupuestos familiares, pero también la asistencia sanitaria construyeron una relación con las familias encuestadas, cruzando los límites de lo privado y registrando los hogares en una trama de escritura en la que pueden leerse los rasgos de una domesticidad entendida como deseable. Las características de los espacios interiores, sus artefactos, pero también los modos de obtención de recursos y la distribución de las tareas al interior del hogar, la adquisición y preparación de los alimentos, sumada a la características de la protección por la vivienda dan forma a la configuración doméstica singular arriba descripta.

Cabe destacar la Tercera Conferencia Internacional sobre la Nutrición como un acontecimiento clave para la conformación de una red de expertos regionales sobre el tema, cuyas múltiples aristas implicaban la necesidad de establecer un diagnóstico a la vez compartido y específico, entre países en situaciones muy disimiles en cuanto a las capacidades estatales y la disponibilidad de información. La presentación del reporte sobre la situación Argentina ante los expertos aquí reunidos por parte el Dr. Escudero, supone no sólo una transmisión de los resultado de la investigación realizada, sino la traducción de las sugerencias de los impulsores de la encuestas a nivel internacional a las particularidades locales y la circulación de los datos específicos en una extensa red de saberes técnicos y formación.

Entendemos que esta experiencia de construcción de inteligibilidad por parte de un organismo estatal sobre un conjunto de familias obreras aporta al campo de estudios sobre domesticidad, familia y hogares obreros un registro de las prácticas cotidianas de alimentación novedosa en su profundidad y detalle, estableciendo un interesante cruce de los debates acerca de las condiciones de vida, los salarios obreros, la división sexual del trabajo, y la consideración del hogar como ámbito de intervención estatal. Todos estos elementos constituyen claves de lectura fundamentales para el estudio de la expansión del consumo de los sectores asalariados producido en la década subsiguiente. 


\section{Bibliografía}

AGUILAR, Paula Lucía, El hogar como problema y como solución. Una mirada genealógica de la domesticidad a través de las politicas sociales. Argentina 1890-1940, Ediciones del Centro Cultural de la Cooperación, Buenos Aires, 2014.

BALLENT, Anahí y GORELIK, Adrián, "País urbano o país rural: la modernización territorial y su crisis”, en CATARUZZA, Alejandro (director) Nueva Historia Argentina, T. VII, Los años treinta, Sudamericana, Buenos Aires, 2002, pp. 143-200.

BIGWOOD, Édouard Jean, Guiding principles for Studies on the Nutrition of Populations, League of Nations Health organization, Technical commission on Nutrition, Geneva, Columbia University Press, New York, 1939.

BUNGE, Alejandro, "Formación del sentido económico de la Mujer", en La economía Argentina. La conciencia Nacional y el problema económico, V. 1, Agencia General de Librerías y Publicaciones, Buenos Aires, 1928.

CALDO, Paula, "Recetas, ecónomas, marcas y publicidades: la educación de las mujeres cocineras de la sociedad de consumo (Argentina, 1920-1945)”, en Arenal, Granada, 2013, V. 20, No 1, pp. 159-190.

ELENA, Eduardo, Dignifying Argentina. Peronism, citizenship, and mass consumption, University of Pittsburgh Press, Pittsburgh, 2011.

GONZALEZ BOLLO, Hernán, "Ciencias Sociales y Sociografía Estatal. Tras el estudio de la familia obrera”, en Estudios Sociales, Santa Fe, 1999, Año IX, pp. 19-39.

GONZALEZ BOLLO, Hernán, La fábrica de las cifras oficiales del Estado Argentino (1869-1947), Universidad Nacional de Quilmes, Bernal, 2014.

GONZALEZ BOLLO, Hernán, "La cuestión obrera en números: La estadística socio-laboral argentina y su impacto en la política y la sociedad, 1895-1943”, en OTERO, Hernán (director) El Mosaico Argentino. Modelos y representaciones del espacio y la población, Siglos XIX y XX, Siglo XXI Editores, Buenos Aires, 2004, pp. 331-375.

JOHNSON, David, ROGERS, John y TAN, Lucilla, “A century of family budgets in the United States”, en Monthly Labor Review, Washington, mayo, 2001, pp. 28-45.

LAZARSFELD, Paul, "Afterword. Toward a History of Sociography", en LAZARSFELD, Paul, JAHODA, Marie y ZEIZEL, Hans Marienthal. The Sociograpphy of an unemployed community, Aldine Atherton Inc, Chicago, 1971, p. 99-115.

MARICHAL, Eugenia, Conocimiento cientifico-técnico y regulaciones jurídicas. El control de los alimentos como red: el caso del Código Bromatológico y el Instituto Bromatológico de la Provincia de Santa Fe (1939-1941), Maestría en Ciencia, Tecnología y Sociedad. Universidad Nacional de Quilmes, 2012, mimeo. 
MARSHALL, Adriana, "La composición del consumo de los obreros industriales de Buenos Aires, 19301980”, en Desarrollo Económico, Buenos Aires, 1981, V. XXI, No 83, pp. 351-374.

MILANESIO, Natalia, Cuando los trabajadores salieron de compras. Nuevos consumidores, publicidad y cambio cultural durante el primer peronismo, Siglo XXI Editores, Buenos Aires, 2014.

PELOSI, Hebe Carmen, El Museo Social Argentino y la Universidad del Museo Social Argentino. Historia y Proyección (1911-1978), Universidad del Museo Social Argentino, Buenos Aires, 2000.

PEREZ, Inés, El hogar tecnificado. Familias, género y vida cotidiana (1940-1970), Editorial Biblos, Buenos Aires, 2012.

PERNET, Corine, "Developing Nutritional Standards and Food Policy: Latin American Reformers between the ILO, the League of Nations Health Organization, and the Pan-American Sanitary Bureau”, en KOTT, Sandrine y DROUX, Joëlle, Globalizing Social Rights: The International Labor Organization and Beyond, Palgrave Mc Millan, New York, 2013 pp. 249-261.

PESEZ, Jean Marie, "Historia de la cultura material", en DE LAS TRAVIESAS MORENO, Luis y ALONSO GONZALEZ, Gladys (editores) La Historia y el oficio de historiador: Colectivo de autores francés, Editorial de Ciencias Sociales , La Habana, 1996, pp. 191-224.

RAMACCIOTTI, Karina, La politica sanitaria del peronismo, Buenos Aires, Biblos, 2009.

ROCCHI, Fernando, "Inventando la soberanía del consumidor: publicidad, privacidad y revolución en Argentina 1860-1940”, en DEVOTO, Fernando y MADERO, Marta (directores) Historia de la vida privada en la Argentina. T. II: La Argentina plural, 1870-1930, Taurus, Buenos Aires 1999, pp. 301-321.

SANCHEZ, María Inés, El espacio doméstico en Buenos Aires (1872-1935): Concepciones, modelos e imaginarios, Librería Concentra, Buenos Aires, 2010.

WEINDLING, Paul, "Social medicine at the League of Nations Health Organization and the International Labor Office compared”, en WEINDLING, Paul (editor) International Health Organizations and Movements (1918-1939), Cambridge University Press, Cambridge, 1995, pp. 134-149.

ZIMMERMAN, Eduardo, Los liberales reformistas. La cuestión social en la Argentina (1890-1916), Buenos Aires, Editorial Sudamericana, 1994. 